\title{
Elastic Strain Tensor Mapping - Extending the Limits of EBSD Analysis
}

\author{
Angus J. Wilkinson*, Graham Meaden** and David J. Dingley*** \\ * Department of Materials, University of Oxford, Parks Road, Oxford OX1 3PH, U.K. \\ ** BLG Productions, 3 Sydenham Road, Bristol, BS6 5SH, U.K. \\ *** Department of Physics, University of Bristol, \& TSL/EDAX Draper, UT 84020, U.S.A.
}

Electron back scatter diffraction (EBSD) is a key quantitative microstructural analysis technique in many commercial and academic materials research laboratories. The typical angular precision of orientation measurements made by commercial EBSD systems is of the order of $0.5^{\circ}$, or $10^{-2} \mathrm{rad}$. With the elastic limit typically at a strain of $\sim 10^{-3}$ this precision needs to be improved by a least an order of magnitude if elastic strain effects are to be detectable.

Small shifts in the positions of zone axes in the EBSD patterns can be detected and measured with sufficient sensitivity to enable strain measurement [1]. In previous work Wilkinson attained a sensitivity of $\pm 2 \times 10^{-4}$ using a large specimen to screen distance, which was used to increase the angular resolution of the EBSD detector at the expense of the large capture angle usually employed for orientation measurements. A general framework to relate pattern shifts measured at four zone axes to the elements of a distortion tensor representing an arbitrary strain and rotation has also been presented [2].

Higher resolution, $1000 \times 1000$ pixel, fast read out CCD cameras, are now available in typical modern EBSD detectors compared to the 578 x 385 pixels slow scan CCD used in Wilkinson's initial work $[1,2]$. This factor coupled with improved algorithms for pattern shift determination now make it possible to establish variations in the entire strain tensor from measurements on individual wide-angle EBSD patterns.

This paper reports on the precision with which pattern shifts can be determined and the resulting strain sensitivity achieved. To this end we report results from three experiments: firstly pattern shifts induced by displacing the incident electron beam, secondly small rigid body rotations applied to the sample, and thirdly strain variations across a $\mathrm{SiGe/Si}$ epilayer viewed in cross section.

In order to induce a uniform shift of the pattern across the scintillator screen the electron beam was deflected away from the optic axis of the SEM on a Ge single crystal sample. Patterns were recorded at 8 displaced beam positions at each of two magnifications $x 400$, and $x 4,000.20$ regions of interest (ROI) were centred at points distributed across the scintillator screen and the pattern shift measured at each as a function of beam position. Figure 1 shows a plot of measured pattern shifts against incident beam displacement, combining data for the two magnifications. The pattern shift can be measured to \pm 0.05 pixels, which at the pattern centre corresponds to an angular uncertainty of $\pm 1.6 \times 10^{-4}$ radians.

The next sequence of EBSD patterns was obtained as a series of small rigid body rotations were applied to a single crystal Ge sample using the SEM stage controls. Five EBSD patterns were recorded after nominal rotations of $0.0^{\circ}, 0.1^{\circ}, 0.2^{\circ}, 0.3^{\circ}, 0.4^{\circ}$, and $0.5^{\circ}$ about the incident beam direction (the y axis). Pattern shifts were determined at four ROI using patterns from the initial 


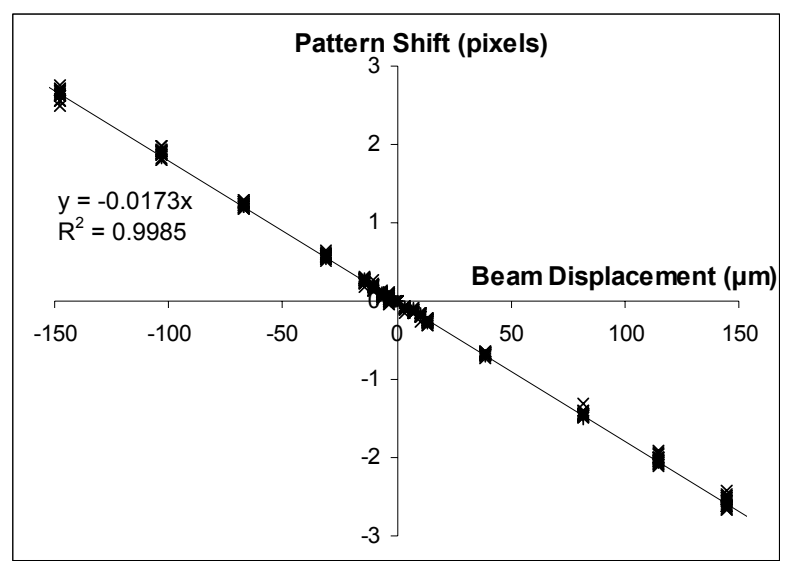

Figure 1: Pattern shifts induced by displacement of incident beam

$' 0.0^{\circ}$ ' set as a reference (zero strain and rotation). These pattern shifts were used to determine the components of the tensor $\mathrm{e}_{\mathrm{ij}}=\mathrm{du}_{\mathrm{j}} / \mathrm{dx}_{\mathrm{i}}$ describing strain and rigid body rotations using the simple linear equations described in [2]. Figure 2 shows that the $\mathrm{du}_{3} / \mathrm{dx}_{1}$ and $\mathrm{du}_{1} / \mathrm{dx}_{3}$ components increase linearly with applied nominal rotations while other components remain small. The magnitudes and opposite signs of $d_{3} / \mathrm{dx}_{1}$ and $\mathrm{du}_{1} / \mathrm{dx}_{3}$ is in accord with the rotation applied nominally about the $y$ axis. Of more importance is the extent of scatter in the results. The standard deviation in measured components of $e_{i j}$ is independent of the nominal rotation angle, but does vary between components (see table 1). This is due to the specific arrangement of the ROI with respect to the axes of the reference frame. On the average the standard deviation is $1.3 \times 10^{-4}$.

The final set of results come from a $\sim 1 \mu \mathrm{m}$ thick $\mathrm{Si}_{1-\mathrm{x}} \mathrm{Ge}_{\mathrm{x}}$ epilayer grown on (001) Si and viewed in cross section. EBSD patterns were obtained every $10 \mathrm{~nm}$ along a scan perpendicular to the interface across the epilayer and $\sim 3.5 \mu \mathrm{m}$ into the substrate. Figure 3 shows the most significant components in the strain variation obtained, here $\mathrm{w}_{31}$ is the asymmetric part of the tensor (rotation), and $\mathrm{e}_{31}$ the symmetric part (shear strain). Relaxation of the tetragonal distortion in the epilayer due to the free surface induces significant curvature and deformation. This occurs not only within the epilayer but also into a significant depth of the substrate.

[1] A. J. Wilkinson, Ultramicroscopy 62 (1996), p. 237.

[2] A. J. Wilkinson, Measuring Strains Using Electron Backscatter Diffraction. In: A. J. Schwartz; M. Kumar, B. L. Adams, Editors, Electron Backscatter Diffraction in Materials Science, Kluwer Academic/Plenum Publishers, Dordrecht, New York, Boston, London (2000). 